\title{
Satellite Cross-Talk Impact Analysis in Airborne Interferometric Global Navigation Satellite System-Reflectometry with the Microwave Interferometric Reflectometer
}

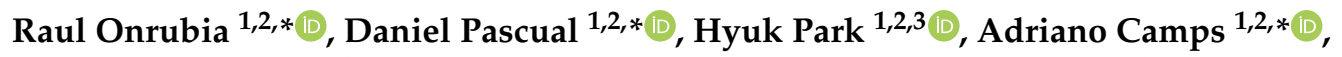 \\ Christoph Rüdiger ${ }^{4}\left(\mathbb{D}\right.$, Jeffrey P. Walker ${ }^{4}(-)$ and Alessandra Monerris ${ }^{5}(\mathbb{C}$ \\ 1 CommSensLab_-Unidad de Excelencia María de Maeztu, Department of Signal Theory and \\ Communications (TSC), Universitat Politècnica de Catalunya-BarcelonaTech (UPC), Jordi Girona 1-3, \\ Campus Nord, D4 Building, 08034 Barcelona, Spain; park.hyuk@tsc.upc.edu \\ 2 Institut d'Estudis Espacials de Catalunya (IEEC/UPC), 08034 Barcelona, Spain \\ 3 Physics Department, School of Telecommunications and Aerospace Engineering (EETAC), \\ Universitat Politècnica de Catalunya-BarcelonaTech (UPC), 08860 Castelldefels, Spain \\ 4 Department of Civil Engineering, Monash University, Clayton, VIC 3800, Australia; \\ chris.rudiger@monash.edu (C.R.); Jeff.Walker@monash.edu (J.P.W.) \\ 5 Department of Infrastructure Engineering, The University of Melbourne, Parkville, VIC 3010, Australia; \\ alessandra.monerris@unimelb.edu.au \\ * Correspondence: onrubia@tsc.upc.edu (R.O.); daniel.pascual@tsc.upc.edu (D.P.); camps@tsc.upc.edu (A.C.); \\ Tel.: +34-93-4054153 (A.C.)
}

Received: 10 April 2019; Accepted: 8 May 2019; Published: 10 May 2019

check for updates

\begin{abstract}
This work analyzes the satellite cross-talk observed by the microwave interferometric reflectometer (MIR), a new global navigation satellite system (GNSS) reflectometer, during an airborne field campaign in Victoria and New South Wales, Australia. MIR is a GNSS reflectometer with two 19-element, dual-band arrays, each of them having four steerable beams. The data collected during the experiment, the characterization of the arrays, and the global positioning system (GPS) and Galileo ephemeris were used to compute the expected delays and power levels of all incoming signals, and the probability of cross-talk was then evaluated. Despite the MIR highly directive arrays, the largest ever for a GNSS-R instrument, one of the flights was found to be contaminated by cross-talk almost half of the time at the L1/E1 frequency band, and all four flights were contaminated $\sim 5-10 \%$ of the time at the L5/E5a frequency band. The cross-talk introduces an error of up to $40 \mathrm{~cm}$ of standard deviation for altimetric applications and about $0.24 \mathrm{~dB}$ for scatterometric applications.
\end{abstract}

Keywords: GNSS; reflectometry; interferometric; satellite cross-talk; antenna array

\section{Introduction}

As compared to conventional global navigation satellite system (GNSS)-reflectometry (cGNSS-R) [1], in which the reflected signal is cross-correlated with a locally generated clean replica of the transmitted code, in interferometric GNSS-R (iGNSS-R) the direct signal transmitted by the GNSS satellites is cross-correlated with the same transmitted signal after it has been scattered on the Earth's surface [2]. This way, iGNSS-R is able to make use of the private signals, that have a wider bandwidth (such as M-code, with up to $40 \mathrm{MHz}$ ), achieving thus a higher accuracy. However, the resulting cross-correlations are noisier than in CGNSS-R, they are more sensitive to radio frequency interferences (RFI), and the cross-correlation peaks of multiple visible satellites may be visible in the correlated data. This last phenomenon is called satellite cross-talk, and affects altimetric and scatterometric 
retrievals [3]. In iGNSS-R, the position of the cross-correlation peak depends on the time difference and the Doppler frequency difference between the direct and the reflected signals. The time delay difference depends on the geometry of the reflections [4]. In ground-based and airborne iGNSS-R, the Doppler difference is essentially non-existent [3]. Since the time delay between the direct and reflected signals is proportional to the sensor height, as altitude decreases the risk of cross-talk increases [3]. This is the case in ground-based and airborne reflectometry as compared to spaceborne reflectometry, and thus high-directive arrays are required to spatially filter the contributions of undesired satellites. In spaceborne reflectometry the direct and reflected signals are received with a different Doppler frequency, but the correlations are also spread in the Doppler domain [5], which in addition to the higher altitude makes cross-talk less frequent but not negligible [3]. Besides, as more constellations are deployed with shared bands (e.g., GPS L1 and Galileo E1, or GPS L5 and Galileo E5a), the probability of two satellites being close in the sky (from the receiver point of view) increases, and so the efficiency of spatial filtering decreases.

This work analyzes the satellite cross-talk that affected four flights of the microwave interferometric reflectometer (MIR) instrument [6]. First, Section 2 briefly describes the MIR instrument and the field campaign. Then, Section 3 describes the two metrics used to analyze the satellite cross-talk, the angular distance to the closest satellite and the worst received signal-to-interference ratio (SIR), and how the cross-talk induced error is estimated. Section 4 presents the results of the analysis, before conclusions are drawn in Section 5.

\section{Instrument and Field Campaign Description}

MIR is a GNSS reflectometer that mimics some of the passive reflectometry and interferometry system-in orbit demonstrator (PARIS-IoD) capabilities [4], such as high directive steerable arrays: two 19-element hexagonal arrays, an up-looking right hand circular polarization (RHCP) one for the direct signal transmitted from the GNSS satellites, and a down-looking left hand circular polarization (LHCP) one for the reflected signal scattered on the Earth's surface. Both antenna arrays are dual-band (L1/E1 and L5/E5a frequency bands), and multi-beam (two beams per band, therefore up to four different satellites can be tracked simultaneously). The received data is sampled by pairs of up- and down-looking beams synchronously at 1 bit for the I and Q components at 32,736 Msps, and stored for later post-processing. A detailed description of the instrument can be found in [6]. The instrument was mounted in a research airplane (see Figure 1).

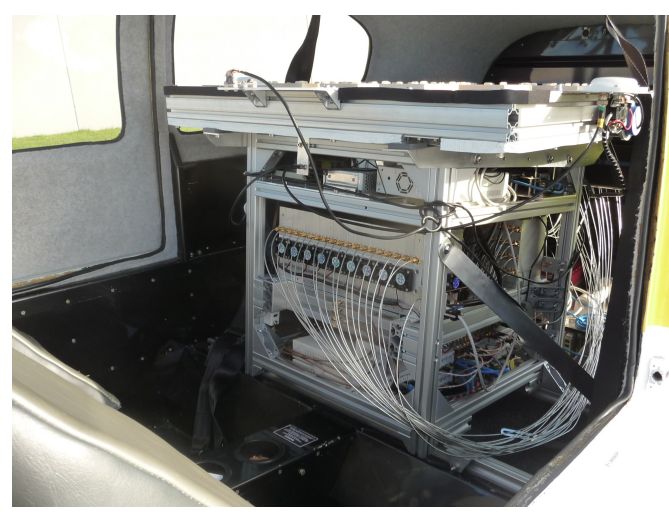

(a)
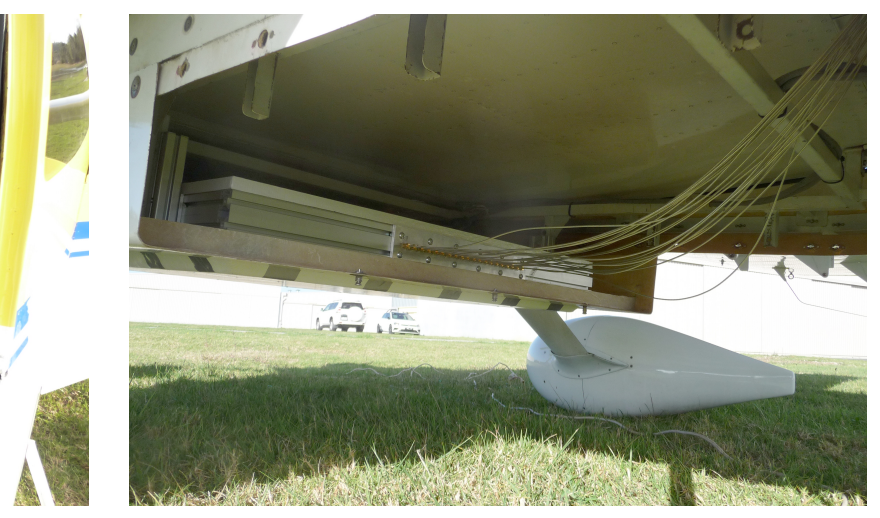

(b)

Figure 1. (a) Microwave interferometric reflectometer (MIR) instrument and up-looking array mounted inside the airplane, and (b) down-looking array covered with a radome hanging from the airplane's fuselage (with part of the fairing removed).

The field campaign was carried out in Victoria and New South Wales, Australia, between April and June 2018. A total of four flights were conducted: two flights over the sea, and two flights over 
land. The first flight was on 30 April 2018 over the entrance to Port Phillip Bay, Victoria, to test the instrument over open water. The airplane flew twice over the Point Lonsdale tide gauge and over the Point Nepean buoy, first at 800 and then at $1500 \mathrm{~m}$ above sea level, and at an average ground speed of $65 \mathrm{~m} / \mathrm{s}$. These stations provided sea level ground truth, and the information on the significant wave height and wave period. The second flight, over the Bass Strait, was conducted on 6 June 2018 departing from Mallacoota (Victoria) following a Cryosat-2 ground track to a distance of $125 \mathrm{~km}$ offshore (see Figure 2). The average ground speed was $75 \mathrm{~m} / \mathrm{s}$ when flying out and $60 \mathrm{~m} / \mathrm{s}$ when flying back, and was flown at $1400 \mathrm{~m}$ above the sea level. The two flights over land were conducted on 1 May 2018 (after 15 days without any rain event, and $9.2 \mathrm{~mm}$ accumulated rainfall in the previous 89 days) and on 18 June 2018 (after $8.2 \mathrm{~mm}$ accumulated rainfall the previous day and $24.6 \mathrm{~mm}$ accumulated rainfall in the previous nine days) [7] over the OzNet soil moisture sensor network in Yanco, NSW [8]. Both flights were conducted at 250-350 $\mathrm{m}$ above the sea level with an average ground speed of $60 \mathrm{~m} / \mathrm{s}$. A total of six hours and $30 \mathrm{~min}$ of data was collected for all four flights.

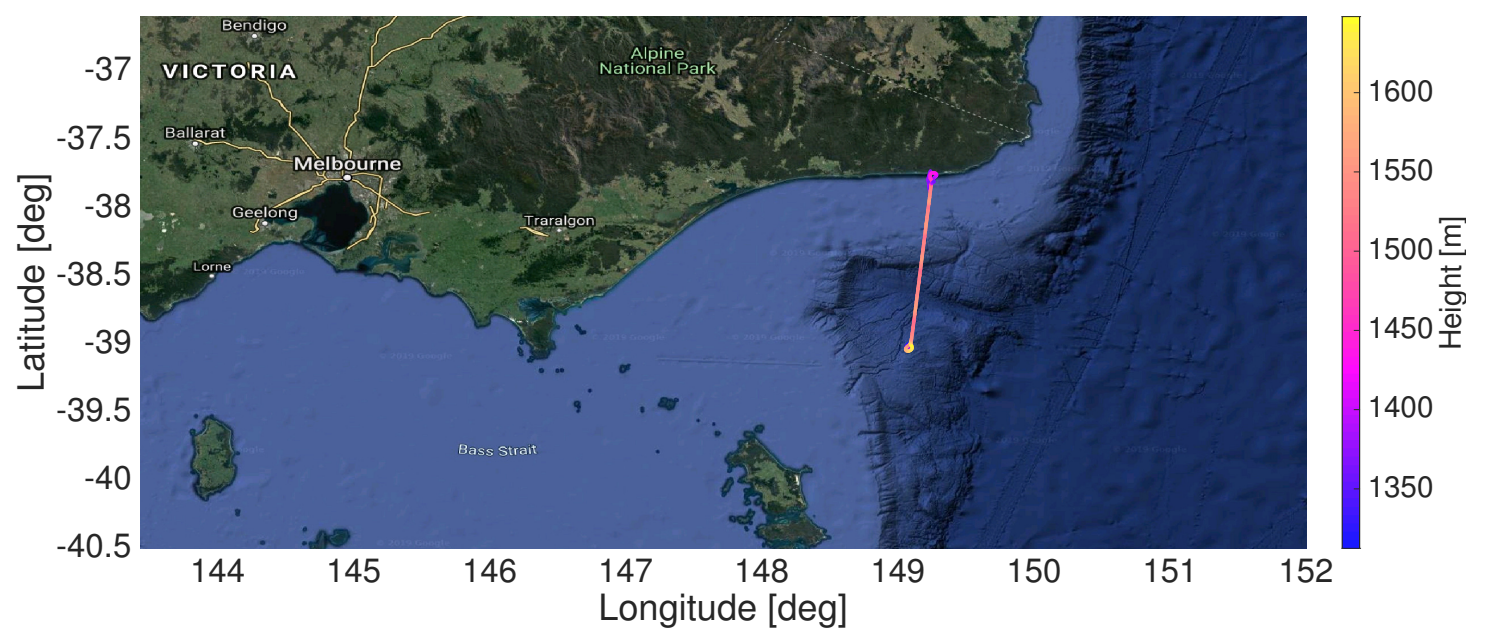

Figure 2. Ground track of sea flight over the Bass Strait. The color scale represents the flight height above the sea level.

According to [3], the MIR highly directive arrays ( $\sim 19 \mathrm{~dB})$ should be able to avoid most of the satellite cross-talk. However, more than half of the Bass Strait flight data was contaminated in the L5/E5a frequency band, which prompted an analysis to determine the instance when this occurred and its impact on the GNSS-R observables.

\section{Methodology}

During the flights, the position, attitude, tracked satellites, and the pointing angles of the instrument were logged. The tracked satellites were chosen based on their elevation angle (the higher the better) in order to maximize the array directivity and to minimize the side lobes. The MIR instrument attempted to track a GPS and a Galileo satellite in each band whenever possible. In case this was not possible, the two satellites with the highest elevation angle were chosen [6].

The angular distance between the tracked satellites and the closest ones was computed using the position of the GPS and Galileo satellites derived from the satellite ephemeris [9]. The expected received power from both the direct and reflected signals from all satellites was simulated using the measured radiation pattern of one of the antenna elements, the array factor according to the array dimensions, and the attitude of the plane. In order to simplify the processing, flat Earth and constant reflectivity was considered for all reflected signals. For each satellite, the delay $\Delta \tau$ between the direct and the reflected signal was approximated using

$$
\Delta \tau=\frac{2 \cdot h \cdot \sin (\theta)}{c}
$$


where $h$ is the height of the plane over the ellipsoid, $\theta$ is the elevation angle of the satellite with respect to the receiver, and $c$ is the speed of light. This delay determines where the centre of the cross-correlation peak should be in the delay domain. The cross-correlation waveforms can be approximated by a two code chips wide triangle in the delay domain in specular reflections [1], one in the leading edge and one in the trailing edge. When the signal is scattered on a rough surface, the trailing edge is elongated. For instance, wind speeds from $6 \mathrm{~m} / \mathrm{s}$ to $22 \mathrm{~m} / \mathrm{s}$ can enlarge the trailing edge of reflections over sea from two to three code chips in airborne GNSS-R [10]. For simplicity, all cross-talk cross-correlation peaks farther than two code chips and weaker than $-10 \mathrm{~dB}$ compared to the tracked one are considered non-interfering.

Figure 3 shows an example of an interferometric contaminated waveform in blue retrieved during the Bass Strait flight, the conventional waveform of the tracked satellite in red, and the waveform of the interfering satellite in yellow. A large number of interferometric waveforms were found to be corrupted due to RFI as well, so they had to be discarded for the study the cross-talk impact assessment. Besides, the interferometric waveform is more affected by noise than the conventional ones, which is used as reference for the comparison because they are not affected by satellite cross-talk. For this reason, this work proposes below how to reconstruct the interferometric waveform using the conventional ones as can be seen in purple in Figure 3.

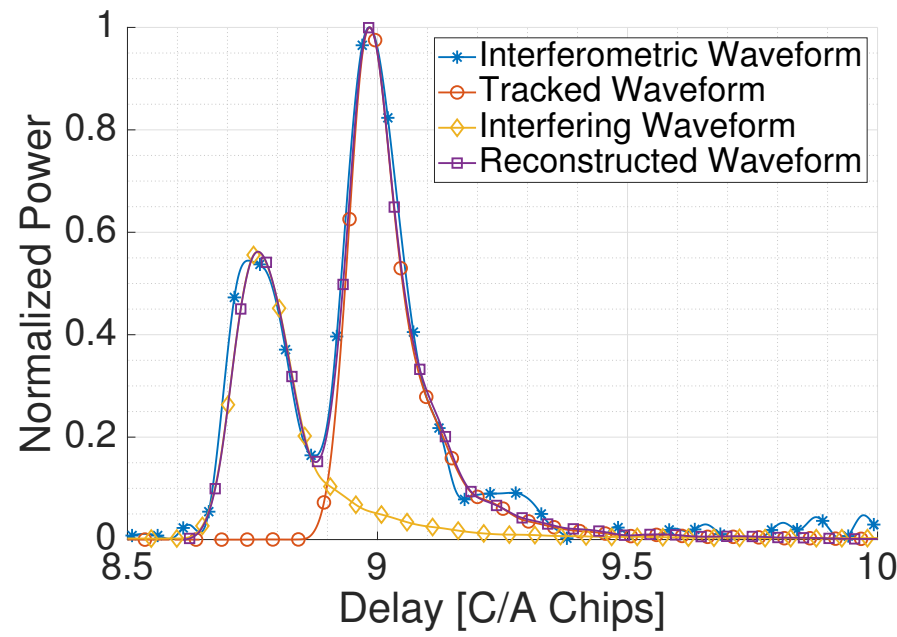

Figure 3. Retrieved L5/E5a interferometric global navigation satellite system-reflectometry (iGNSS-R) waveform (blue), Galileo E5a tracked conventional global navigation satellite system-reflectometry (cGNSS-R) waveform (red), global positioning system (GPS) L5 interfering cGNSS-R waveform (yellow), and reconstructed contaminated waveform (purple). The data was collected during the flight over the Bass Strait.

The coherent waveform between the signals $x$ and $y$ as function of the delay $\tau$ and Doppler frequency $f_{d}$ between them is defined in [5] as

$$
Y_{x, y}\left(t_{0}, \tau, f_{d}\right)=\int_{t_{0}}^{t_{0}+T_{c}} x\left(t_{0}+t^{\prime}\right) \cdot y\left(t_{0}+t^{\prime}+\tau\right) \cdot \exp \left(j 2 \pi f_{d} t^{\prime}\right) d t^{\prime},
$$

where $t_{0}$ is the time at the origin, and $T_{\mathcal{C}}$ is the coherent integration time. Then, the incoherent average of $M$ coherent waveforms is defined as

$$
<\left|Y\left(\tau, f_{d}\right)\right|^{2}>=\frac{1}{M} \cdot \sum_{m=0}^{M-1}\left|Y\left(m \cdot T_{c}, \tau, f_{d}\right)\right|^{2} .
$$

For GNSS signals, the cross-correlation peak is located at a Doppler frequency $f_{d}$ that compensates the Doppler frequency difference between $x$ and $y$, and it is located at a delay $\tau$ that compensates the 
time difference between $x$ and $y$. In the conventional case, when a signal $s$ transmitted from a GNSS satellite with a noise $n$ is cross-correlated with the code $c$ corresponding to the transmitting satellite, the incoherent waveform becomes

$$
<\left|Y\left(\tau, f_{d}\right)\right|^{2}>=<\left|Y_{s, c}\left(\tau, f_{d}\right)+Y_{n, c}\left(\tau, f_{d}\right)\right|^{2}>
$$

where $Y_{s, c}$ stands for the waveform resulting from cross-correlating the received signal $s$ with the code $c$, and $Y_{n, c}$ is the noise term resulting from cross-correlating the noise $n$ with the code $c$. In the interferometric case, if the signal $s$ transmitted from the tracked satellite with noise $n$ is received in conjunction with a signal $z$ transmitted from a satellite which causes cross-talk, the incoherent waveform becomes

$$
\begin{aligned}
<\left|Y\left(\tau, f_{d}\right)\right|^{2}>= & <\mid Y_{s_{D}, s_{R}}\left(\tau, f_{d}\right)+Y_{s_{D}, z_{R}}\left(\tau, f_{d}\right)+Y_{s_{D}, n_{R}}\left(\tau, f_{d}\right)+Y_{z_{D}, s_{R}}\left(\tau, f_{d}\right)+Y_{z_{D}, z_{R}}\left(\tau, f_{d}\right) \\
& +Y_{z_{D}, n_{R}}\left(\tau, f_{d}\right)+Y_{n_{D}, s_{R}}\left(\tau, f_{d}\right)+Y_{n_{D}, z_{R}}\left(\tau, f_{d}\right)+\left.Y_{n_{D}, n_{R}}\left(\tau, f_{d}\right)\right|^{2}>
\end{aligned}
$$

where the subscripts $D$ and $R$ indicate if the signal has been received through the direct or the reflected antenna arrays, respectively, $Y_{S_{D}, s_{R}}$ is the desired satellite term, $Y_{z_{D}, z_{R}}$ is the cross-talk term, $Y_{S_{D}, z_{R}}$ and $Y_{z_{D}, s_{R}}$ are the so called cross-satellite terms, and $Y_{s_{D}, n_{R}}, Y_{z_{D}, n_{R}}, Y_{n_{D}, s_{R}}, Y_{n_{D}, z_{R}}$, and $Y_{n_{D}, n_{R}}$ are the noise terms. For ground-based and airborne GNSS reflectometry, the Doppler difference between the direct and the reflected GNSS signals can be neglected, so the peak of $Y_{s_{D}, s_{R}}$ and $Y_{z_{D}, z_{R}}$ is located at $f_{d}=0$. Due to the low power of the GNSS signals, the term $Y_{n_{D}, n_{R}}$ dominates the other noise terms [11]. If an interference signal $i$ is present, several cross terms appear and might dominate over the desired $Y_{s_{D}, s_{R}}$ and $Y_{z_{D}, z_{R}}$ terms, which happened during most of the flight over the Bass Strait. The interference terms must be avoided, so the contaminated waveform is reconstructed as

$$
\begin{aligned}
<\left|Y_{R E C}(\tau)\right|^{2}>= & <\left|Y_{u_{R}, c_{s}}\left(\tau, f_{s}\right)+Y_{u_{R}, c_{z}}\left(\tau, f_{z}\right)\right|^{2}> \\
= & <\mid Y_{s_{R}, c_{s}}\left(\tau, f_{s}\right)+Y_{z_{R}, c_{s}}\left(\tau, f_{s}\right)+Y_{i_{R}, c_{s}}\left(\tau, f_{s}\right)+Y_{n_{R}, c_{s}}\left(\tau, f_{s}\right) \\
& +Y_{s_{R}, c_{z}}\left(\tau, f_{z}\right)+Y_{z_{R}, c_{z}}\left(\tau, f_{z}\right)+Y_{i_{R}, c_{z}}\left(\tau, f_{z}\right)+\left.Y_{n_{R}, c_{z}}\left(\tau, f_{z}\right)\right|^{2}>,
\end{aligned}
$$

where $u_{R}=s_{R}+z_{R}+i_{R}+n_{R}$ is the signal received from the down-looking array, $s_{R}$ is the received signal from the tracked satellite, $z_{R}$ is the received signal from the satellite causing cross-talk, $i_{R}$ is an interfering signal, and $n_{R}$ is noise; $c_{S}$ and $c_{z}$ are the codes of the desired satellite and the satellite causing cross-talk respectively, and $f_{s}$ and $f_{z}$ are the Doppler frequencies of the satellite being tracked and the satellite causing cross-talk. The transmitted GNSS codes are robust against RFI and noise, which implies that $Y_{s_{R}, c_{s}}$ and $Y_{z_{R}, c_{z}}$ dominate over the other terms, contributing thus as a much lower noise floor compared to the interferometric case. An example of a reconstructed contaminated waveform is shown in Figure 3. The cross-correlation peaks are not symmetric respect to their maximum-that is, the trailing edge is longer than the leading one. This effect is caused by the scattering of the reflected signal in a rough surface, in this case the sea surface roughness caused by the wind [5]. For this reason, the "second" cross-correlation peak (the contribution of the satellite that is received later) is more affected by the "first" one (the contribution of the satellite that is received sooner) than vice versa.

Two datasets are created from the Bass Strait flight data: the XT (cross-talk) dataset with $670 \mathrm{~s}$, and the NOXT (no cross-talk) data set with $470 \mathrm{~s}$. The flights over land were not suitable for this analysis because the soil permittivity would have attenuated some of the tracked and interfering waveforms, affecting the impact analysis statistics. The Port Phillip Bay flight was not suitable because the aeroplane was repeatedly flying over two points of interest, therefore manoeuvring constantly and abruptly, which caused the apparition of even larger side lobes that would had also affected the analysis statistics. In the XT dataset, the two beams of the instrument were pointing at two different satellites that were close enough in the sky to be received by the main lobe of both beams. The signals of both satellites were received in both beams and close enough in time to be overlapped. In one of the beams, the interfering cross-correlation peak was received before the tracked cross-correlation peak, 
and in the other beam it was received afterwards. In the NOXT dataset, both beams were tracking two different satellites that again were received in both beams, but the cross-correlation peaks were separated enough not to be overlapped, thus not being affected by the cross-talk. In the case of XT dataset, the interfering cross-correlation peak and the noise distort the tracked cross-correlation peak, introducing thus an error in any retrieved parameter such as the peak position or amplitude. In order to assess the impact of the cross-talk alone, the impact of the noise has also to be characterized, using for that purpose the NOXT data set.

In the altimetric case, the analysis focused on the three main delay estimators: the position of the maximum derivative (DER) [12], the position of the maximum amplitude (MAX) [12,13], and the position when the leading edge reached the $75 \%$ of the maximum amplitude as proposed by [14], which is called half-power position (HALF) due to its similarity to the homonym estimator used by the radar community. The delay positions using MAX, DER, and HALF are estimated for the reconstructed and the tracked conventional waveforms. For each estimator, the error between the estimated positions was computed between both waveforms. Then, the results for the XT and NOXT data sets were compared in terms of their probability density function (PDF). In the NOXT dataset, the tracked and the interfering cross-correlation peaks are not overlapping each other. For a given delay estimator, the estimated delay difference between the conventional and the reconstructed waveforms might be only caused by the different noise terms. The PDF of the NOXT term was used as reference. In the XT dataset, the tracked and the interfering cross-correlation peaks were overlapping each other, therefore the PDFs might be different to the NOXT ones. These PDFs were then compared to assess the impact of the cross-talk.

To evaluate the impact in scatterometry, the same analysis is applied to the peak amplitude difference between the reconstructed and the conventional waveforms.

The reconstructed contaminated waveform $\left\langle\left|Y_{R E C}\right|^{2}>\right.$ and the conventional waveforms of both the interfering $<\left|Y_{z_{R}, c_{z}}\right|^{2}>$ and the tracked satellites $<\left|Y_{s_{R}, c_{s}}\right|^{2}>$ were computed with $T_{\mathcal{C}}=1 \mathrm{~ms}$ coherent averaging and $M=1000$ incoherent averages, and then interpolated to reach $1 \mathrm{~cm}$ resolution around the peaks and in the leading edges.

While the cross-talk occurrence has been analyzed in both frequency bands, the cross-talk impact in altimetry and scatterometry has been analyzed only at the L5/E5a frequency band. This is because the L5/E5a beam is wider than the L1/E1 one, which allows the simultaneous reception of signals from more than one satellite that were not overlapping in time, so it was possible to have both XT and NOXT datasets. Furthermore, GPS L5 signals and Galileo E5a signals were similar in modulation, bandwidth, and chip length, so their correlation peaks are similar. This fact simplified the analysis, since just two cases have to be analyzed: the tracked satellite cross-correlation peak being located before or after the interfering satellite cross-correlation peak.

\section{Results}

Figure 4 shows skyplots of the satellites in view (black traces). The tracked satellites are represented with coloured dots, where the colour represents the angular distance to the closest satellite. In case the tracked satellite was affected by cross-talk it is also highlighted in red. Note that the four beams are represented in the same plot, and if the same satellite was tracked in both bands their representations are overlapped, which may under-represent the visual cross-talk occurrence.

Figure 5 shows the cumulative histogram of the cross-talk probability for a given signal-to-interference ratio (SIR), which is the relationship between the expected power of the tracked satellite over the expected power of the other satellites. The histogram was computed taking into account the lowest SIR from all visible satellites, since satellite cross-talk did not occur from more than one satellite simultaneously. The cross-talk occurrence of the two beams at each frequency band was aggregated if the beams were not pointing to the same satellite - that is, the data from the second beam was considered as if the flight was longer. 


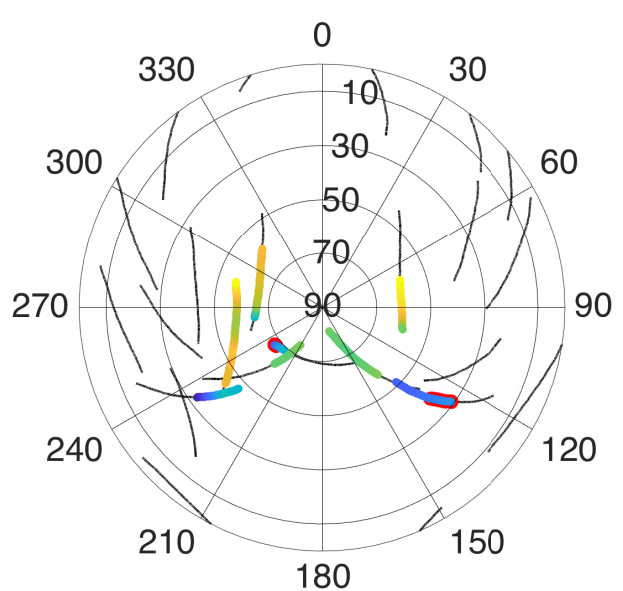

(a)

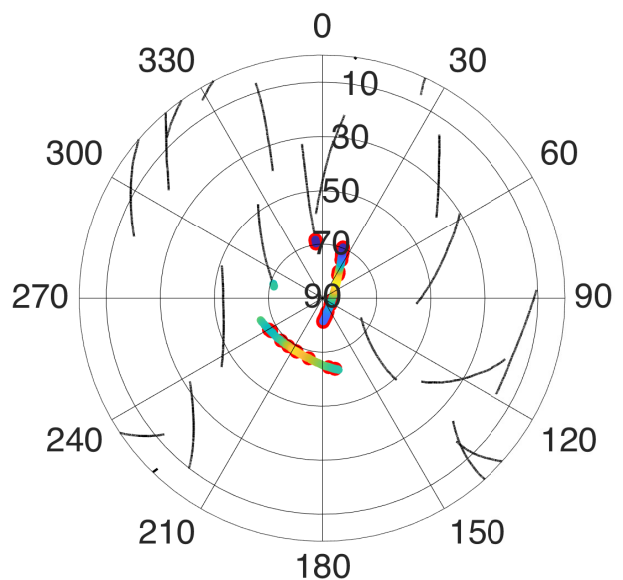

(c)

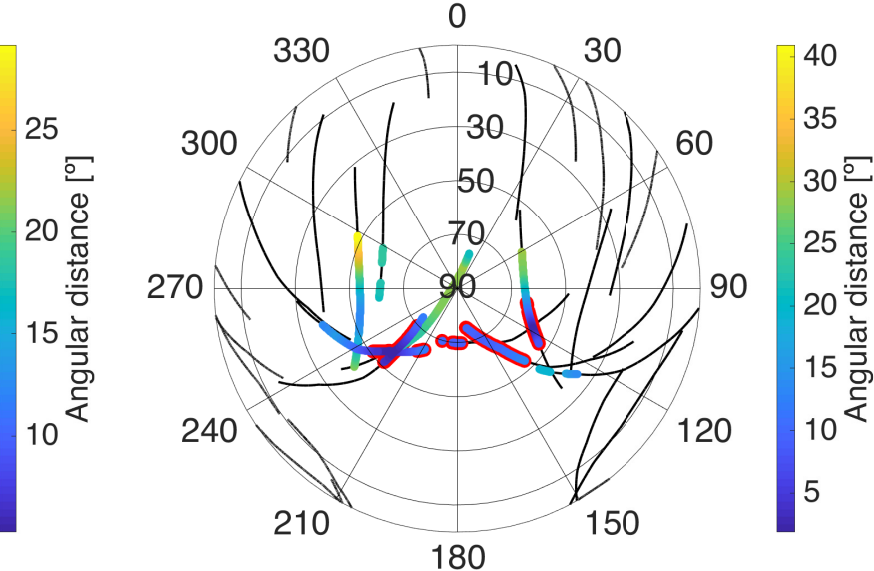

(b)
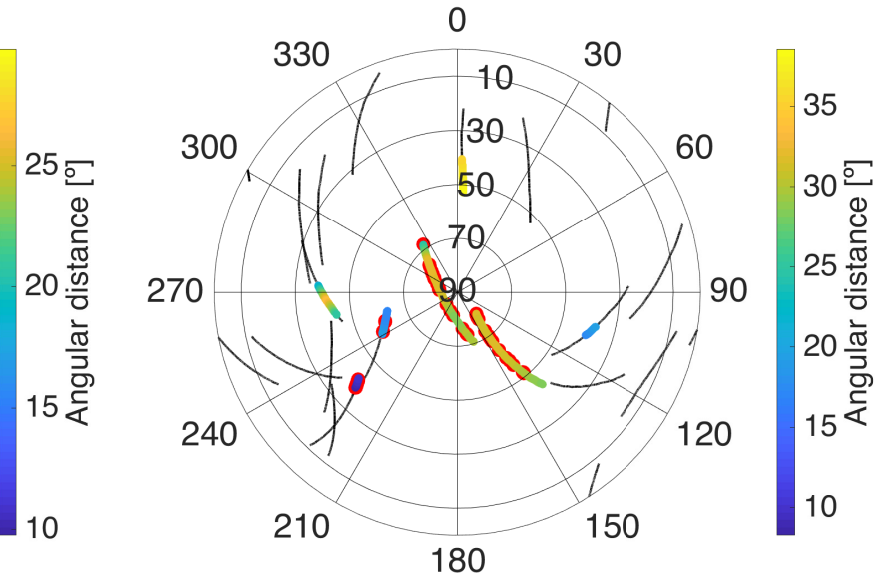

(d)

Figure 4. Skyplot of the tracked satellites in the (a) flight over the entrance to Port Philip Bay, (b) sea flight over the Bass Strait, and flights over Yanco under dry (c) and wet (d) soil conditions. The color scale indicates the angular distance to the closest satellite. Red lines highlight cross-talk from other satellites.

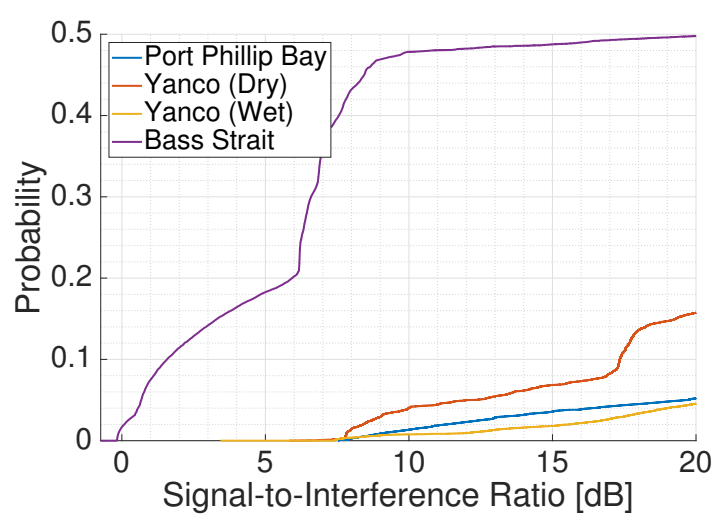

(a)

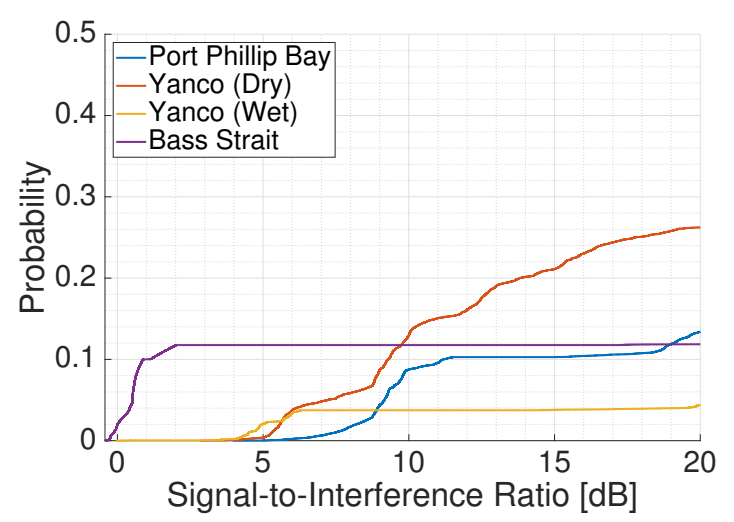

(b)

Figure 5. Cumulative histogram of the probability of having cross-talk in the (a) L1/E1 and, (b) L5/E5a frequency bands for the different MIR flights. 
Several factors determine the likelihood to be affected by satellite cross-talk. First, the number of GNSS satellites transmitting in-band: L1/E1 seems a priori more likely to be affected because these signals were transmitted by the full GPS constellation (32 satellites) and Galileo constellation (16 satellites at the time of the experiment), while the signals allocated in L5/E5a were only transmitted by the GPS block IIF satellites (12 of the 32 current GPS satellites), plus the Galileo ones (16 satellites). Secondly, the L1/E1 transmitted signals were more susceptible to cross-talk because the chip length was 10 times longer than in L5/E5, which means that the range of elevation angles that might interfere according to Equation (1) was larger. Finally, the array factor at L1/E1 had a narrower beam as compared to the one at L5/E5a, which was more selective against the undesired signals, but the long separation between antenna elements $\left(0.75 \lambda_{L 1}\right.$, where $\left.\lambda_{L 1}=19.04 \mathrm{~cm}\right)$ caused diffraction lobes as the instrument pointed far away from the boresight, which happened often when the airplane rotated heavily about the pitch and roll axes. This effect can be clearly seen in the flights over land. In the skyplots in Figure 4 the cross-talk is intermittent, which was caused by the diffraction lobes generated when the airplane had to manoeuvre abruptly at the end of each transect over the farm fields.

The position of the satellites in the sky during the flights had a strong impact in the probability of having cross-talk because of their short duration. Counterintuitively, L1/E1 signals show much lower likelihood of being affected by cross-talk than the L5/E5a one, except during the second sea flight, which was contaminated almost half of the flight time. In the other flights, the probability of cross-talk was reduced to less than 2\%. L5/E5a signals were instead more likely to be affected by cross-talk $(5-10 \%)$. Since there are fewer satellites transmitting L5/E5a signals, there is a lower chance of finding one of them with a high elevation angle. The MIR instrument was therefore forced to track satellites with lower elevation angles, and therefore the cross-talk probability increases [3]. It was noteworthy that not even a 19-element array antenna with 18-20 dB directivity has been able to avoid a cross-talk contamination during half of one of the flights, and that it could not reduce the cross-talk at L5/E5a below $10 \%$.

Figure 6 shows the error PDF when estimating (a) the maximum derivative point, (b) the half power point, (c) the peak position, and (d) the peak amplitude. The "NOXT" PDFs show the error estimation caused only by the noise. The "XT" PDFs show the error estimation caused by satellite cross-talk and the noise. The analysis distinguishes between the interfering cross-correlation peak arriving after the tracked satellite cross-correlation peak ("first peak"), or arriving before ("second peak"). The mean and standard deviation of each PDF is shown in Table 1. In the case of the amplitude, the mean and standard deviation are first estimated as the geometric mean and geometric standard deviation, and then they are converted into decibels. The position error is considered positive if the point position is delayed, and the amplitude error is considered positive if the amplitude increases.

When the interfering cross-correlation peak was received after the tracked satellite one ("first peak"), the standard deviation of the estimations slightly increased with respect to the NOXT case, but the bias was not affected significantly. Since the delay estimators were applied to the leading edge of the first peak, the interfering one has to be really close to affect these estimators-that is, being almost totally overlapping. Furthermore, the symmetry of the PDF and the unchanged bias suggests that the PDF has changed only because the reconstructed waveform (see Equation (6)) has more noise terms that the conventional one (see Equation (4)).

On the contrary, when the interfering cross-correlation peak was received before the tracked satellite one ("second peak"), the PDF lost its symmetry, it was biased, and the standard deviation was increased up to $40 \mathrm{~cm}$ with respect to the previous case. The PDFs show the DER point moving in the opposite direction than the HALF and the MAX points, as expected. The trailing edge of the interfering cross-correlation peak advanced in time with the MAX and the HALF positions because the trailing edge can be approximated by a decreasing exponential function $f(\tau)=e^{-k \cdot \tau}$, which is a monotonic decreasing function. On the other hand, the DER point was delayed because the derivative of the trailing edge $f^{\prime}(\tau)=-k \cdot e^{-k \cdot \tau}$ is a monotonic increasing function. The maximum derivative delay estimator seemed the more robust one, whereas the half estimator was the most affected by cross-talk. 
All the estimators showed an increase of the standard deviation up to tenths of $\mathrm{cm}$, which prevents the desired centimetric precision in altimetric applications [4]. The peak value estimation seems to be affected in the same way as the delay estimators; if the interfering cross-correlation peak was received after the tracked satellite cross-correlation peak, the amplitude estimation was not affected, whereas in the opposite case, the PDF was biased and the std increased up to $0.24 \mathrm{~dB}$, which would affect the accuracy of retrieved geophysical parameters, such as soil moisture.

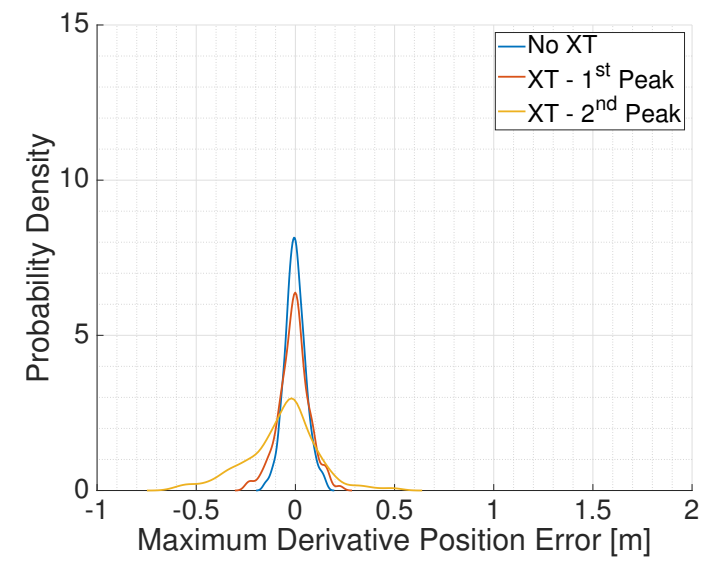

(a)

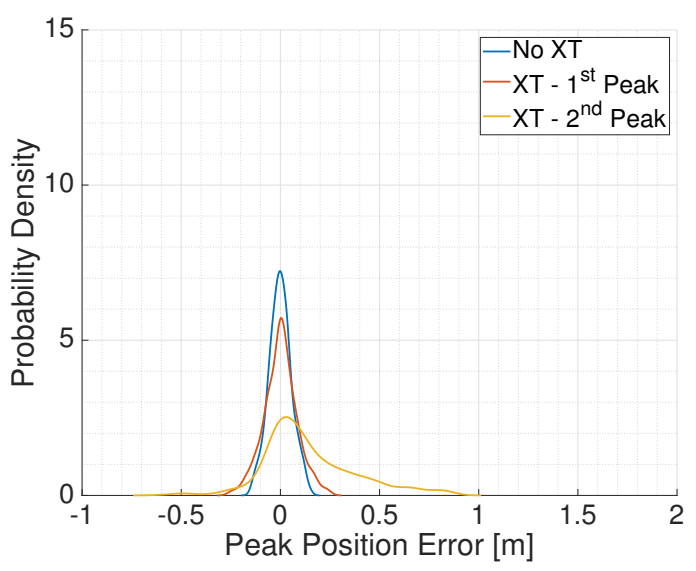

(c)

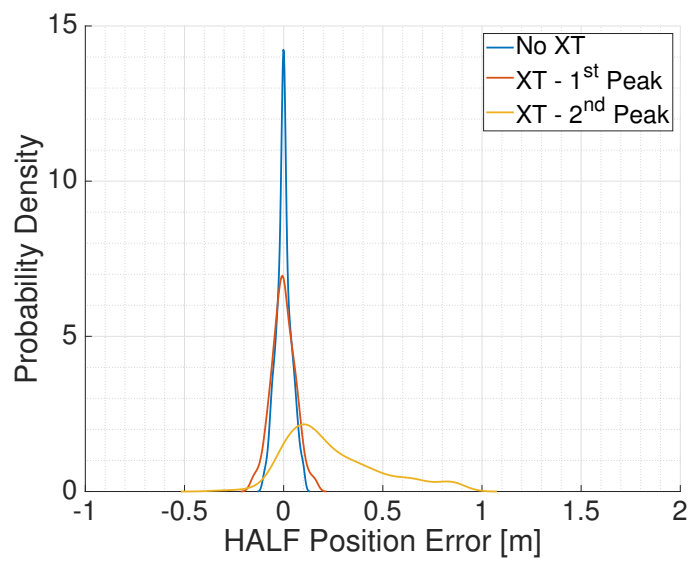

(b)

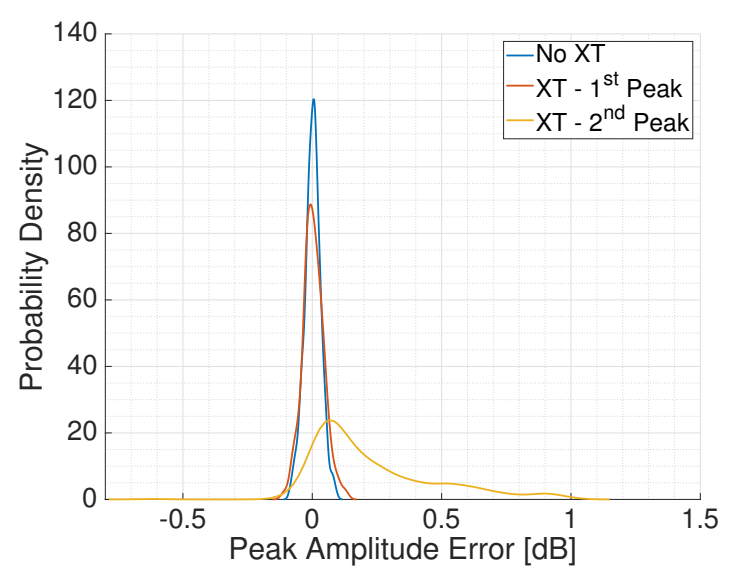

(d)

Figure 6. Probability density function (PDF) of the estimation error of the (a) maximum derivative position (DER), (b) half-power position (HALF), (c) peak position (MAX), and (d) peak amplitude. The "XT" PDFs show the error estimation when the cross-correlation peaks are close enough to interfere with each other, while the "NOXT" show the error estimation when the cross-correlation peaks are far enough not to interfere. The analysis distinguishes between the interfering cross-correlation peak arriving later than the tracked satellite cross-correlation peak ("first peak"), or arriving sooner ("second peak"). 
Table 1. Effect of cross-talk in three different delay estimators and in the peak amplitude in the flight over the Bass Strait.

\begin{tabular}{cccc}
\hline & \multirow{2}{*}{ No XT } & \multicolumn{2}{c}{ XT } \\
\cline { 2 - 4 } & & 1st Peak & 2nd Peak \\
\hline \multirow{2}{*}{ MAX [cm] } & $\mu=-0.14$ & $\mu=0$ & $\mu=14.4$ \\
& $\sigma=5.6$ & $\sigma=8.4$ & $\sigma=23.5$ \\
\hline \multirow{2}{*}{ DER [cm] } & $\mu=-0.1$ & $\mu=-0.8$ & $\mu=-6.9$ \\
& $\sigma=5.3$ & $\sigma=7.8$ & $\sigma=18.1$ \\
\hline \multirow{2}{*}{ HALF $[\mathrm{cm}]$} & $\mu=-0.1$ & $\mu=-0.4$ & $\mu=40.1$ \\
& $\sigma=4.2$ & $\sigma=6.4$ & $\sigma=45$ \\
\hline \multirow{2}{*}{ Peak value $[\mathrm{dB}]$} & $\mu=0$ & $\mu=0$ & $\mu=-0.2$ \\
& $\sigma=0.03$ & $\sigma=0.04$ & $\sigma=0.24$ \\
\hline
\end{tabular}

\section{Conclusions}

This work shows evidence of satellite cross-talk in iGNSS-R using highly directive antennas, and analyzes its impact on three different delay estimators and the peak value estimation. While this effect should be less important for spaceborne receivers [3], its effects are still not negligible and must be accounted for. The cross-talk probabilities for different signal-to-interference ratios have been computed and contaminated waveforms have been presented. The cross-talk occurrence below $10 \%$ of the total time in three of the four flights are in accordance with the predictions in [3], but longer flights are recommended to validate it.

The effect of cross-talk in the main three delay estimators (MAX, DER, and HALF) and in the peak amplitude estimation has also been assessed for this particular flight and sea surface roughness. The standard deviation of the error estimation increased from $18 \mathrm{~cm}$ up to $40 \mathrm{~cm}$, which exceeds the centimetric precision of the altimetric accuracy requirements. Analogously, the standard deviation of the amplitude estimation increased up to $0.24 \mathrm{~dB}$, which also might affect the retrievals of soil parameters such as surface roughness and soil moisture. Even using highly directive arrays, the degradation of the waveforms caused by satellite cross-talk could not be avoided, which should be taken into account for future ground-based and airborne interferometric GNSS-R missions. Results may differ for other sea conditions, and further studies are required both over sea and land.

Author Contributions: Conceptualization, R.O., D.P., H.P. and A.C.; methodology, R.O.; software, R.O.; validation, R.O. and D.P.; formal analysis, R.O.; investigation, R.O. and D.P.; resources, H.P., A.C., C.R., J.P.W. and A.M.; data curation, R.O. and D.P.; writing-original draft preparation, R.O.; writing-review and editing, R.O., D.P., H.P., A.C., C.R., J.P.W. and A.M.; visualization, R.O. and D.P.; supervision, H.P., A.C., C.R., J.P.W. and A.M.; project administration, A.C.; funding acquisition, A.C.

Funding: This work was supported by the Spanish Ministry of Economy and Competitiveness and FEDER EU under the project "AGORA: Técnicas Avanzadas en Teledetección Aplicada Usando Señales GNSS y Otras Señales de Oportunidad" (MINECO/FEDER) ESP2015-70014-C2-1-R by the Agencia Estatal de Investigación, and by the Spanish Ministry of Science, Innovation and Universities, "Sensing with Pioneering Opportunistic Techniques", grant RTI2018-099008-B-C21, and the grant for recruitment of early-stage research staff FI-DGR 2015 of the AGAUR - Generalitat de Catalunya (FEDER), Spain, and Unidad de Excelencia María de Maeztu MDM-2016-0600, and to Prof. Camps ICREA Academia 2015 award of the Generalitat de Catalunya.

Acknowledgments: Thanks to Tomoji Takasu for his help finding a system unified ephemeris database.

Conflicts of Interest: The authors declare no conflict of interest.

\section{Abbreviations}

The following abbreviations are used in this manuscript:

CDF cummulative distribution function

cGNSS-R conventional global navigation satellite systems-reflectometry

GNSS global navigation satellite systems

GNSS-R global navigation satellite systems-reflectometry

GPS global positioning system 


$\begin{array}{ll}\text { iGNSS-R } & \text { interferometric global navigation satellite systems-reflectometry } \\ \text { MIR } & \text { microwave interferometric reflectometer } \\ \text { NOXT } & \text { no cross-talk dataset } \\ \text { PARIS-IoD } & \text { passive reflectometry and interferometry system-in orbit demonstrator } \\ \text { PDF } & \text { probability density function } \\ \text { RFI } & \text { radio frequency interference } \\ \text { SIR } & \text { signal-to-interference ratio } \\ \text { XT } & \text { cross-talk dataset }\end{array}$

\section{References}

1. Zavorotny, V.U.; Gleason, S.; Cardellach, E.; Camps, A. Tutorial on remote sensing using GNSS bistatic radar of opportunity. IEEE Geosci. Remote Sens. Mag. 2014, 2, 8-45.10.1109/MGRS.2014.2374220. [CrossRef]

2. Martin-Neira, M.; D'Addio, S.; Buck, C.; Floury, N.; Prieto-Cerdeira, R. The PARIS Ocean Altimeter In-Orbit Demonstrator. IEEE Trans. Geosci. Remote Sens. 2011, 49, 2209-2237.10.1109/TGRS.2010.2092431. [CrossRef]

3. Pascual, D.; Park, H.; Onrubia, R.; Arroyo, A.A.; Querol, J.; Camps, A. Crosstalk Statistics and Impact in Interferometric GNSS-R. IEEE J. Sel. Top. Appl. Earth Obs. Remote Sens. 2016, 9, 4621-4630.10.1109/JSTARS.2016.2551981. [CrossRef]

4. Martin-Neira, M.; Caparrini, M.; Font-Rossello, J.; Lannelongue, S.; Vallmitjana, C. The PARIS concept: An experimental demonstration of sea surface altimetry using GPS reflected signals. IEEE Trans. Geosci. Remote Sens. 2001, 39, 142-150.10.1109/36.898676. [CrossRef]

5. Zavorotny, V.; Voronovich, A. Scattering of GPS signals from the ocean with wind remote sensing application. IEEE Trans. Geosci. Remote Sens. 2000, 38, 951-964.10.1109/36.841977. [CrossRef]

6. Onrubia, R.; Pascual, D.; Querol, J.; Park, H.; Camps, A. The Global Navigation Satellite Systems Reflectometry (GNSS-R) Microwave Interferometric Reflectometer: Hardware, Calibration, and Validation Experiments. Sensors 2019, 19, 1019.10.3390/s19051019. [CrossRef] [PubMed]

7. Daily Rainfall-Narrandera Airport AWS-Bureau of Meteorology. Available online: http://www.bom.gov. $\mathrm{au} / \mathrm{jsp} / \mathrm{ncc} / \mathrm{cdio} /$ weatherData/av?p_nccObsCode=136\&p_display_type=dailyDataFile\&p_startYear= 2018\&p_c=-1099598755\&p_stn_num=074148 (accessed on 8 April 2019).

8. Smith, A.B.; Walker, J.P.; Western, A.W.; Young, R.I.; Ellett, K.M.; Pipunic, R.C.; Grayson, R.B.; Siriwardena, L.; Chiew, F.H.S.; Richter, H. The Murrumbidgee soil moisture monitoring network data set. Water Resour. Res. 2012, 48.10.1029/2012WR011976. [CrossRef]

9. The Multi-GNSS Experiment and Pilot Project (MGEX). Available online: http://mgex.igs.org/ (accessed on 28 January 2019).

10. Rodriguez-Alvarez, N.; Akos, D.M.; Zavorotny, V.U.; Smith, J.A.; Camps, A.; Fairall, C.W. Airborne GNSS-R Wind Retrievals Using Delay-Doppler Maps. IEEE Trans. Geosci. Remote Sens. 2013, 51, 626-641.10.1109/TGRS.2012.2196437. [CrossRef]

11. Querol, J.; Alonso-Arroyo, A.; Onrubia, R.; Pascual, D.; Park, H.; Camps, A. SNR Degradation in GNSS-R Measurements Under the Effects of Radio-Frequency Interference. IEEE J. Sel. Top. Appl. Earth Obs. Remote Sens. 2016, 9, 4865-4878.10.1109/JSTARS.2016.2597438. [CrossRef]

12. Rius, A.; Cardellach, E.; Martin-Neira, M. Altimetric Analysis of the Sea-Surface GPS-Reflected Signals. IEEE Trans. Geosci. Remote Sens. 2010, 48, 2119-2127.10.1109/TGRS.2009.2036721. [CrossRef]

13. Hofmann-Wellenhof, B.; Lichtenegger, H.; Wasle, E. GNSS Global Navigation Satellite Systems; GPS, Glonass, Galileo and More; Springer: Wien, Austria; New York, NY, USA, 2008; p. 96.

14. Cardellach, E.; Rius, A.; Martin-Neira, M.; Fabra, F.; Nogues-Correig, O.; Ribo, S.; Kainulainen, J.; Camps, A.; D'Addio, S. Consolidating the Precision of Interferometric GNSS-R Ocean Altimetry Using Airborne Experimental Data. IEEE Trans. Geosci. Remote Sens. 2014, 52, 4992-5004.10.1109/TGRS.2013.2286257. [CrossRef]

(C) 2019 by the authors. Licensee MDPI, Basel, Switzerland. This article is an open access article distributed under the terms and conditions of the Creative Commons Attribution (CC BY) license (http:// creativecommons.org/licenses/by/4.0/). 